\title{
Una experiencia de aprendizaje-servicio en futuros docentes: desarrollo de la competencia social y ciudadana
}

\section{A service-learning experience in future teachers: development of the social and civic competence}

\author{
Jesús GIL-GÓMEZ ${ }^{1}$, Odet MOLINER-GARCÍA ${ }^{1}$, Óscar CHIVA-BARTOLL ${ }^{2}$ y \\ †Rafaela GARCÍA LÓPEZ ${ }^{2}$ \\ ${ }^{1}$ Universitat Jaume I y ${ }^{2}$ Universitat de Valencia
}

Recibido: Marzo 2014

Evaluado: Septiembre 2014

Aceptado: Octubre 2014

\section{Resumen}

Este artículo presenta los resultados de aplicar un programa de ApS a estudiantes de maestro/a en el ámbito de la Educación Física. En concreto se analiza su incidencia en el desarrollo de la competencia social y ciudadana, desde una perspectiva metodológica mixta. La complementariedad cuantitativa-cualitativa plantea un marco adecuado de aproximación al objeto de estudio. Cuantitativamente la investigación muestra tendencias de mejora en algunas dimensiones (Conformidad con lo socialmente correcto, Sensibilidad social, Ayuda y colaboración y Responsabilidad Social) que son refrendadas en el análisis cualitativo. A partir del objetivo planteado en la investigación y vistos los datos obtenidos y su discusión, se concluye que el ApS aplicado en el ámbito de la formación de futuros docentes es una buena herramienta para desarrollar su competencia social y ciudadana.

Palabras clave: aprendizaje-servicio, metodología, educación cívica, educación superior, Educación Física, formación de profesores.

\begin{abstract}
This paper presents the results of applying a Service-Learning program in pre-service teachers in the field of PE. The impact in the development of the social and civic competence was analyzed using a mixed method approach. The quantitative-qualitative complementarity offered the appropriate framework to approach the object of study. Our quantitative results showed some trends of improvement in some dimensions (Agreement in the Socially Established as Correct Beliefs, Social Sensitivity, Help and Collaboration and Social Responsibility)which were also reflected in the qualitative analysis. From the target stated, and after having reviewed the obtained data and its discussion, it was concluded that the Service-Learning applied in preservice teachers is a good way to develop the social and civic competence.
\end{abstract}

Keywords: service-learning, methodology, civic education, higher education, physical education, training of teachers 
El EEES ha supuesto, entre otras cuestiones, una oportunidad para la deseable renovación metodológica en las universidades españolas (Calvo-Bernardino y Mingorance-Arnáiz, 2009). En parte, este cambio se concreta en la tendencia a utilizar las denominadas metodologías activas que, junto con la aplicación de un sistema de evaluación continuo o formativo, constituyen los dos grandes pilares de la renovación metodológica universitaria (Palomares, 2011).

Otro de los principios que guían el nuevo planteamiento pedagógico es la búsqueda de la excelencia. En este sentido, existen autores que defienden la inclusión de la formación ciudadana en la educación superior de forma explícita como elemento que confiere realmente excelencia a la formación (Esteban, 2004; García, Escámez, Martínez, M. y Martínez MJ., 2008; García, Gozálvez, Vázquez y Escámez, 2010; Herrero, 2006; Santos y Lorenzo, 2010; Touriñán, 2007). Aun asumiendo que la función prioritaria de las enseñanzas superiores es la profesionalización, la consideran insuficiente, pues es difícil ser un buen profesional sin un compromiso ético asociado a cualquier profesión, y sin un compromiso responsable con la sociedad, con su mejora y con el bien común. Desde este planteamiento y dada la compleja situación social en que vivimos, la educación ciudadana se ha convertido en una necesidad acuciante (Print, 2003).

La definición de competencia ha sido un tema complejo, aunque podemos decir que existe consenso sobre sus características básicas: implica conocimientos, habilidades y actitudes y conductas integrados entre sí, incluye capacidades informales y de procedimiento y supone aprendizaje continuo unido a la experiencia y a la capacidad de ponerse en acción (Bisquerra y Pérez, 2007). Estos mismos autores clasifican las competencias en dos grandes grupos: las técnico-profesionales y las socio-personales. Dentro del segundo grupo podemos ubicar la competencia básica social y ciudadana objeto de nuestro estudio. El Ministerio de Educación y Ciencia ${ }^{1}$ la define como la capacidad de comprender la realidad social en que se vive, de cooperar, convivir y ejercer la ciudadanía democrática en una sociedad plural, así como comprometerse a su mejora. A partir de esta conceptualización, Pagès (2009) determina las habilidades y actitudes que dan cuerpo a esta competencia. Entre las habilidades destacan la de comunicarse, mostrar tolerancia, expresar y comprender puntos de vista diferentes, negociar inspirando confianza, gestionar el estrés y la frustración, interactuar eficazmente en el ámbito público, manifestar solidaridad e interés por resolver problemas que afectan a la comunidad, reflexionar críticamente y participar constructivamente. Las actitudes incluyen la colaboración y seguridad en uno mismo, el interés por el desarrollo socioeconómico, la comunicación intercultural, la diversidad de valores y el respeto a los demás, la superación de prejuicios, el compromiso, el sentido de pertenencia y de responsabilidad, el apoyo a la diversidad y la voluntad de respetar los valores de los demás.

Sin duda, la competencia social y ciudadana es amplia y compleja. En la tabla 1, a efectos de medición, la sintetizamos a partir de las seis dimensiones del cuestionario

\footnotetext{
${ }^{1}$ RD 1513/2006
} 
validado por Moraleda, González Galán y García-Gallo (2004), a las que añadimos la idea de ciudadanía activa de Martínez (2006).

\begin{tabular}{l|l}
\hline \multicolumn{1}{c|}{ DIMENSIONES } & \multicolumn{1}{c}{ DEFINICIÓN } \\
\hline $\begin{array}{l}\text { Conformidad con lo } \\
\text { socialmente correcto }\end{array}$ & $\begin{array}{l}\text { Respeto hacia las personas con autoridad en el ámbito académico, aceptación } \\
\text { de las normas de convivencia en casa y respeto hacia las cosas de los } \\
\text { compañeros }\end{array}$ \\
\hline Sensibilidad social & $\begin{array}{l}\text { Empatía hacia otros, capacidad de escuchar, comprender y descubrir } \\
\text { cualidades en los demás, satisfacción por compartir la alegría cuando a otra } \\
\text { persona algo le sale bien y por estar con los compañeros }\end{array}$ \\
\hline Ayuda y colaboración & $\begin{array}{l}\text { Generosidad con los demás, capacidad de trabajar en grupo y de ayudar a los } \\
\text { componentes de un equipo y de dedicar tiempo a los demás si lo necesitan }\end{array}$ \\
\hline Seguridad y firmeza en \\
la interacción & $\begin{array}{l}\text { Capacidad de reconocer errores, defender derechos y de dialogar para } \\
\text { solucionarlo, seguridad en uno mismo y poder discutir cuando se tenga la } \\
\text { sensación de injusticia de forma serena y razonable }\end{array}$ \\
\hline Liderazgo prosocial & $\begin{array}{l}\text { Capacidad de movilizar a los demás, de sugerir y aportar ideas y la } \\
\text { satisfacción por organizar nuevas actividades }\end{array}$ \\
\hline Responsabilidad social & $\begin{array}{l}\text { Implicación personal para actuar ante problemas sociales aceptándolos como } \\
\text { parte de uno y aceptando la responsabilidad individual, capacidad de asumir } \\
\text { obligaciones sin que nadie lo tenga que recordar y de implicarse en proyectos } \\
\text { para mejorar la sociedad }\end{array}$ \\
\hline
\end{tabular}

Tabla 1: Dimensiones de la Competencia Social y Ciudadana. Adaptada de Moraleda, González Galán y García-Gallo (2004).

Una vez hecha esta aproximación conceptual, la pregunta a la que pretende responder este trabajo es: ¿existe alguna metodología que cumpla los requisitos de ser calificada como activa y que permita a su vez la formación ciudadana? Nuestra respuesta es sí: el Aprendizaje-Servicio (ApS en adelante). Su fundamento es aprender contenidos curriculares a la vez que se presta un servicio a la sociedad atendiendo una necesidad real no cubierta, desde una perspectiva de colaboración recíproca (Puig, Gijón, Martín y Rubio, 2011). El ApS traza un puente entre el mundo académico y el social, de forma que con su uso abordamos la creación de buenos ciudadanos, de profesionales con ética y con un amplio sentido de participación social.

Tal y como hemos dicho, nos encontramos en un momento en el que es necesaria una transición pedagógica que adecúe el mundo educativo a los cambios acaecidos en la sociedad. En este sentido, Riera y Civís (2008) plantean algunos retos que se deben abordar. Uno de ellos se refiere a la incorporación de una nueva pedagogía comunitaria a través de la creación de redes socio-educativas que complementen la formación escolar. El ApS responde a esta necesidad, incorporando instituciones y lugares sociales al hecho educativo. Asimismo, permite pasar del conocimiento disciplinar y disciplinario al conocimiento complejo y responsabilizador, dando valor a desarrollar 
entre el alumnado la capacidad de autocrítica, de seleccionar la información y dotarla de significado, de reconocer y respetar la diversidad existente y, en fin, de profundizar en la ética común del género humano, siendo éste otro de los retos importantes a afrontar según estos autores. En consecuencia, el ApS se nos muestra como una herramienta adecuada para enfocar la deseada innovación pedagógica desde las premisas expuestas.

El ApS puede llegar a ser un rasgo distintivo de la organización educativa que lo promueva y utilice, contribuyendo a la formación integral de las personas y a la definición del paradigma escolar que el centro adopte, de acuerdo con Santos (2013). De los cuatro vigentes en el sistema educativo español, según Penalva (2009), el ApS contribuye a la formación de una escuela ubicada en su conceptualización como comunidad de participación o como comunidad creadora de significados, las cuales ofrecen, en nuestra opinión, mejores posibilidades para introducir paralelamente a la formación académica, la formación en las denominadas competencias socio-personales (Bisquerra y Pérez, 2007) entra las que se encuentra la social y ciudadana objeto de esta investigación.

En el campo de la Educación Física (en adelante EF) la innovación educativa también se abre camino. Barbosa-Rinaldi (2008) expresa la necesidad de adoptar prácticas pedagógicas diferentes en $\mathrm{EF}$, en las que el papel del docente sea de mediador del conocimiento, posibilitando la reflexión del alumnado y desarrollando su sentido crítico acerca del mundo en el que vive. En esta línea, Cremasco y Castellani (2012) destacan las posibilidades y la idoneidad de la EF para desarrollar una ciudadanía caracterizada por la confirmación y ampliación de los derechos colectivos (cívicos, políticos y sociales). Los antecedentes expuestos ofrecen indicios de la aptitud de la EF para el desarrollo de la ciudadanía. Nuestra investigación abunda en este tema desde la innovación metodológica en la EF a través del ApS, avanzando en un campo en que, si bien existen estudios sobre sus efectos generales (Conway, Amel y Gerwien, 2009; Yorio y Feifei, 2012), son limitadas las investigaciones en este ámbito.

A nivel nacional destacan los trabajos de Ríos $(2003,2009)$ sobre el uso de esta metodología en la integración de discapacitados y de población reclusa. Internacionalmente, la revisión de Cervantes y Meaney (2003) indica que la investigación de los efectos del ApS en EF es un tema pendiente. En este sentido, Himelein, Passman y Phillips (2010) y Massey-Sokes y Meaney (2006) realizaron aplicaciones de APS en futuros docentes para actuar sobre la prevención de la obesidad infantil en EEUU; La Master (2001) lo aplicó en escuelas de una zona con graves conflictos sociales y económicos; y Domangue y Carson (2008) lo hicieron realizando actividades físicas para niños y niñas que abandonaron sus hogares y se instalaron en un asentamiento temporal a causa del huracán Katrina. En todos estos estudios se reportan efectos beneficiosos del uso de esta metodología en la formación de futuros docentes de EF.

Este artículo presenta los resultados de aplicar un programa formativo a estudiantes de maestro/a mediante ApS en el ámbito de la E F, analizando su incidencia en el desarrollo de la competencia social y ciudadana desde un planteamiento metodológico mixto (cuantitativo-cualitativo). 


\section{Método}

Esta investigación sigue un diseño cuasi-experimental que se aborda desde la complementariedad metodológica cualitativa-cuantitativa (mixed methods), muy utilizada en la investigación educativa actual (Anguera, 2011; Anguera, Camerino, Castañer y Sánchez-Algarra, 2014). A partir del paradigma investigador cuantitativo, formulamos una hipótesis causal a contrastar entre grupos equivalentes con grupo de control y medidas prestest-postest. Asimismo, desde el paradigma cualitativo planteamos una pregunta de investigación para complementar los hallazgos cuantitativos. Concretamente se realiza un estudio descriptivo con objeto de aproximarnos y comprender las reflexiones y valoraciones del estudiantado respecto a la experiencia del ApS y a los cambios que se producen en relación al desarrollo de su competencia social y ciudadana. Este planteamiento integrador en la metodología investigadora responde a la realidad compleja que presenta el campo de investigación.

\section{Hipótesis y pregunta de investigación}

La hipótesis cuantitativa es la siguiente: la aplicación del programa de ApS, producirá en el alumnado del grupo experimental una mejora estadísticamente significativa $(\mathrm{p}<0,05)$ en su competencia social y ciudadana que diferirá y será superior a las puntuaciones obtenidas por el grupo de control.

Asimismo, en este artículo pretendemos dar respuesta a la siguiente pregunta de investigación: ¿el programa de ApS sirve para desarrollar la competencia social y ciudadana en el alumnado participante?

\section{Muestra}

Los participantes han sido 84 estudiantes de la asignatura "Bases anatómicas y Fisiológicas del Movimiento” (curso 2011-12), de la titulación de Maestro de Educación Primaria (Especialidad EF) de la Universitat Jaume I. El 52\% son hombres y el $48 \%$ mujeres, oscilando sus edades entre los 18 y los 33 años. La asignación al grupo experimental $(\mathrm{N}=46)$ y al grupo de control $(\mathrm{N}=38)$ ha sido no probabilística e incidental, atendiendo a consideraciones prácticas. El alumnado del grupo experimental constituyó grupos de intervención de 4-5 componentes.

\section{Instrumentos}

Los datos cuantitativos se recogieron mediante el cuestionario EVCPI (Evaluación de la Competencia Ciudadana Prosocial e Inclusiva) creado "ad hoc" y basado en una adaptación del de Moraleda et al. (2004) en el que se valoran las siguientes dimensiones: conformidad con lo socialmente correcto, sensibilidad social, ayuda y colaboración, seguridad y firmeza en la interacción, liderazgo prosocial y responsabilidad social (ver tabla 1). Se trata de una escala tipo Likert de cinco puntos, en la que el 1 representa "muy en desacuerdo" y el 5 "muy de acuerdo". El 
cuestionario consta de 31 ítems (p.e. "Cuando un compañero/a ha triunfado en algo, me gusta participar de su alegría y felicitarle") y ha sido validado mediante juicio de expertos. La fiabilidad medida mediante el estadístico Alpha de Cronbach es de 0.89, considerada muy alta.La recopilación de datos cualitativos para la investigación se realizó mediante la cumplimentación por parte del alumnado de dos documentos. Para diferenciarlos claramente e identificar la procedencia de la información en su análisis, se les asignaron los acrónimos que se describen a continuación. Al primero lo denominamos Documento de Servicio Asignatura (DSA) y servía para organizar los roles dentro de los grupos y anotar las reflexiones y opiniones sobre la actividad de carácter académico y personal (vivencias, sentimientos...). El segundo (Diario de Seguimiento de Servicio-DSS) servía para profundizar en la reflexión personal, analizar los problemas surgidos y aportar soluciones de cara a futuras sesiones. Una vez cumplimentados debían ser enviados por correo electrónico al profesor, con lo que éste siempre estaba al corriente del punto en que se encontraba cada grupo.

\section{Descripción de la propuesta formativa mediante ApS}

El programa aplicado mediante ApS constituye la variable independiente de nuestra investigación. Se desarrolló durante 11 semanas en el segundo semestre del curso académico (febrero-mayo). El alumnado participante se distribuyó en grupos de 4-5 personas para la prestación del servicio. El programa formativo estaba integrado dentro de la asignatura, siendo una actividad evaluable y calificable, respondiendo a las nuevas directrices metodológicas del EEES. Se desarrolló en las 6 fases que establecen Puig et al. (2007) y las guías de la RMC Research Corporation (2006): planificación, acción, demostración, reflexión, reconocimiento y evaluación.

Se optó por la modalidad de servicio directo, relacionado con la mejora de la movilidad de personas que presentan algún tipo de diversidad funcional ${ }^{2}$. El alumnado diseñaba y aplicaba sesiones de EF dirigidas a niños y niñas que presentaban como característica común una movilidad reducida, con el objetivo de trabajar/aprender contenidos de sistemática del ejercicio, de artrología y de miología. Además, las clases suponían un contacto personal con niños y niñas que tienen limitaciones diarias derivadas de su diversidad, conociendo su realidad cotidiana, sus perspectivas, etc. Los resultados de la ejecución de las sesiones prácticas se vieron día a día, haciéndose patente para el alumnado tanto la utilidad para los receptores como sus propios progresos en conocimientos, habilidades de relación y comprensión del contexto social. Se trabajó con cuatro entidades del entramado social de la ciudad de Castellón de la Plana: Maset de Frater, Fundación Síndrome de Down, Asociación de Diabéticos y Colegio Público Serrano Suñer. En cuanto a la duración del proyecto, cada grupo invirtió 136 horas de trabajo de media, lo que supone una dedicación de 12,3 horas semanales, de las cuales 8 se corresponden con la realización del servicio directo

\footnotetext{
${ }^{2}$ En el artículo se utiliza esta terminología, de acuerdo con los criterios del Foro de Vida Independiente y Divertad. Más información en : http://www.forovidaindependiente.org/node/45
} 
(puesta en práctica de las sesiones). Las demás son horas dedicadas a la preparación, reflexión, tutorías y evaluación de la intervención.

En este punto es necesario dar una pincelada que clarifique la filosofía adoptada en el trato con las personas y entidades receptoras del servicio. La actuación en este colectivo en ningún caso se enfocó desde la caridad, el interés o desde una perspectiva médico-asistencial, sino desde la pedagogía de la autonomía (Vega, 2007), buscando contribuir al desarrollo integral de las personas desde una implicación educativa que llegue más allá del voluntariado o el mero servicio comunitario (Rodríguez, 2014). El seguimiento de las sesiones prácticas que constituyen el programa formativo era supervisado continuamente por el profesorado de la asignatura y por los profesionales de las entidades implicadas. De acuerdo con la metodología ApS, los y las docentes adoptaron el rol de guía, dejando al alumnado el protagonismo en el proceso. No obstante, al presentar la población receptora del servicio problemáticas complejas relacionadas con su diversidad, las sesiones eran supervisadas por el profesorado y los especialistas, buscando el justo punto de equilibrio entre la seguridad total de la actividad y el papel protagonista del alumnado. Asimismo, este proceso se complementó con la realización de reflexiones conjuntas entre el profesor de la asignatura y los grupos de intervención, así como entre todo el grupo-clase, estableciéndose así el necesario sistema de reflexión que debe acompañar a cualquier aplicación de ApS (Puig, Batlle, Bosch y Palos, 2007; RMC Research Corporation, 2006).

\section{Tratamiento de la información}

Los datos cuantitativos se han tratado con la aplicación SPSS 18. Se han realizado contrastes de medias (t de Student) de acuerdo cn el siguiente gráfico.

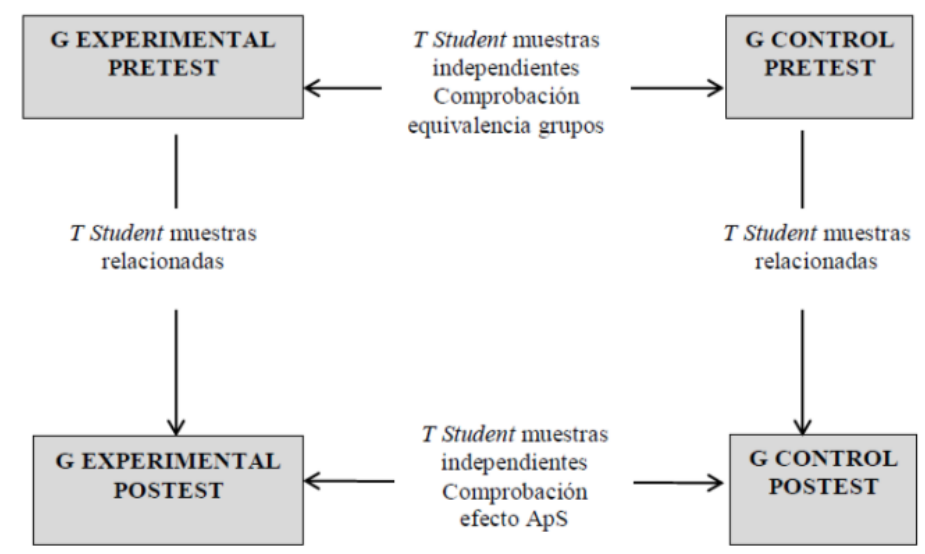

Gráfico 1. Esquema análisis cuantitativo 
Para realizar el análisis cualitativo de la información se ha realizado un análisis de contenido de tipo deductivo. Para ello se establecieron unas categorías iniciales coincidentes con las seis dimensiones de la competencia social y ciudadana establecidas en el cuestionario EVCPI, rediseñando el modelo inicial en función de la revisión del material, lo que supone que emerja el significado de cada sector, párrafo, acontecimiento o situación. Las subcategorías han constituido el modelo final del análisis y para este proceso se utilizó la aplicación informática Nudist Vivo

\section{Resultados}

\section{Resultados cuantitativos}

La comparación de las puntuaciones medias entre los grupos experimental y control en el pretest mostró que no se encontraron diferencias significativas. Se obtuvo un valor $\mathrm{P}>0.05$, por lo que los consideramos equivalentes.

En relación al contraste de medias pretest-postest no se han obtenido diferencias significativas en los grupos experimental y control, obteniendo $p>0.05$ en ambos casos.

Finalmente se contrastaron las diferencias entre los postests de los dos grupos. Se obtuvo $\mathrm{p}>0.05$, por lo que no hay diferencias significativas entre el postest del grupo experimental y del control. El mismo análisis estadístico se ha seguido para contrastar cada una de las seis dimensiones de la variable, no obteniendo significatividad en ninguna comparación. No obstante, en la comparación de los postests de los dos grupos se obtiene una mejora no significativa en cuatro de las seis dimensiones que conforman el constructo competencia social y ciudadana, concretamente en conformidad con lo socialmente correcto, sensibilidad social, ayuda y colaboración y responsabilidad social, obteniendo las dos restantes puntuaciones similares. El gráfico siguiente muestra este efecto. 


\section{Medias}

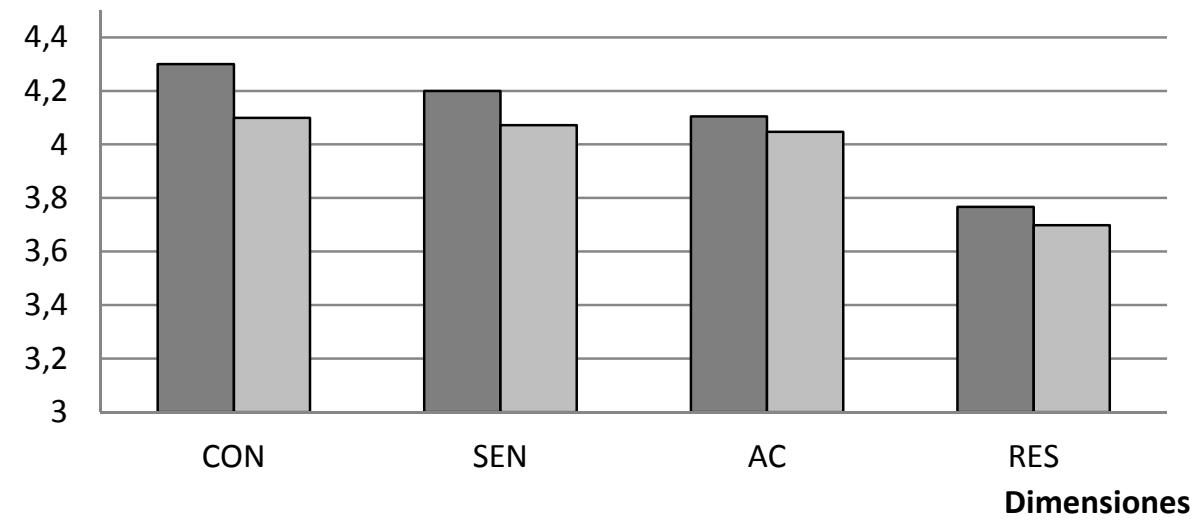

Media postest grupo experimental $\square$ Media postest grupo control

Gráfico 2. Medias postest dimensiones competencia social y ciudadana

El gráfico muestra como las puntuaciones en el postest del grupo experimental de las cuatro dimensiones se sitúan por encima de los valores del postest del grupo control. Estos resultados indican una tendencia en la que se debería profundizar en futuras investigaciones.

\section{Resultados cualitativos}

El modelo inicial de análisis de contenido constaba de seis dimensiones correspondientes a las definidas en el cuestionario cuantitativo. De ellas, en cuatro se han obtenido hallazgos y en dos no ha habido resultados reseñables ("Seguridad y firmeza en la interacción” y "Liderazgo prosocial”). En la siguiente tabla se representan las diferentes categorías y subcategorías obtenidas en el análisis de contenido. 


\begin{tabular}{|c|c|c|c|c|c|}
\hline \multicolumn{6}{|c|}{ CONFORMIDAD CON LO SOCLALMENTE CORRECTO } \\
\hline \multicolumn{6}{|c|}{ SENSIBILIDAD SOCLAL } \\
\hline \multicolumn{2}{|c|}{ Empatía } & \multicolumn{2}{|c|}{ Relación con los demás } & \multicolumn{2}{|c|}{ Consciencia social } \\
\hline $\begin{array}{l}\text { Discriminar } \\
\text { estados de } \\
\text { ánimo }\end{array}$ & $\begin{array}{c}\text { Ponerse en } \\
\text { lugar del otro }\end{array}$ & $\begin{array}{c}\text { Valorar a los } \\
\text { otros }\end{array}$ & $\begin{array}{l}\text { Tener una } \\
\text { imagen } \\
\text { positiva }\end{array}$ & $\begin{array}{l}\text { Descubrir la } \\
\text { realidad social }\end{array}$ & $\begin{array}{l}\text { Implicación } \\
\text { profesional }\end{array}$ \\
\hline \multicolumn{6}{|c|}{ AYUDA Y COLABORACIÓN } \\
\hline \multicolumn{3}{|c|}{ Colaboración alumnado/participantes } & \multicolumn{3}{|c|}{ Trabajo en grupo } \\
\hline \multicolumn{6}{|c|}{ RESPONSABILIDAD SOCLAL } \\
\hline \multicolumn{2}{|c|}{ Propia y profesional } & \multicolumn{2}{|c|}{ Sistema educativo } & \multicolumn{2}{|c|}{ Familia } \\
\hline
\end{tabular}

Tabla 2: Resultados análisis de contenido

A continuación presentamos los resultados intercalando citas textuales que ilustran las subcategorías (nodos) halladas en el análisis.

\section{Categoría "Conformidad con lo socialmente correcto"}

Esta categoría se compone de dos subcategorías: normas sociales y convivencia.

- $\quad$ Normas sociales

Los estudiantes son conscientes de que la ayuda que se puede prestar a personas con necesidades especiales está regulada por determinadas normas, para evitar actuaciones que, a pesar de la buena voluntad, podrían entorpecer más que ayudar. Aluden a la necesidad de conocer y acatar normas que emanan de personas con reconocida autoridad moral. En el trato con alumnado de primaria, que son legalmente menores de edad, los futuros maestros se han dado cuenta de la importancia de acatar y cumplir determinadas normas sociales y legales. Este hecho se hace especialmente relevante en el caso de menores con diversidad funcional.

"Hemos realizado unas cuantas fotos para nuestro power point con el permiso de los coordinadores del centro, padres y el de los propios alumnos." (DSS-G1F5 ${ }^{3}$ )

${ }^{3}$ Como norma general, todas las citas han sido codificadas de acuerdo con las claves DSS (Diario Seguimiento Servicio) o DSA (Documento Servicio Asignatura); G+número indica el grupo de intervención y F+letra/número indica la ficha de procedencia de los datos. 
- Convivencia

Se dan cuenta de que acatar las normas sociales facilita la convivencia y propicia un buen clima de respeto, ayudándoles a integrarse adecuadamente en una organización y establecer relaciones provechosas, tanto a nivel profesional como personal:

"Las sensaciones experimentadas a lo largo de las sesiones han sido muy satisfactorias, ya que además del cariño que nos han expresado los niños, en el centro nos han ayudado muchísimo y nos aceptaron enseguida." (DSS-G5FP)

\section{Categoría "Sensibilidad Social” (SEN)}

Esta categoría se subdivide en: empatía (discriminar estados de ánimo, ponerse en lugar del otro), relación con los demás (valorar a los demás, tener imagen positiva de los demás) y consciencia social (descubrimiento realidad social, implicación profesional). Se describen a continuación.

\section{- Empatía}

La Sensibilidad Social se identifica con la capacidad de ser empático, lo cual implica, por una parte, poder discriminar estados de ánimo en los demás y, por otra, ponerse en lugar del otro.

- Empatía: discriminar estados de ánimo

Sin duda, tener esta capacidad abre el abanico de actuación para un maestro, convirtiéndolo en mejor profesional. En este sentido encontramos evidencias de haber conseguido la adquisición de esta competencia entre el alumnado. Los estudiantes detectan sentimientos que provocan estados de ánimo como la amabilidad, el agradecimiento, la felicidad y el cariño:

"Hemos visto que con tan solo prestar un poco de atención a alguien ya puedes hacerle feliz.” (DSS-G12F1)

- Empatía: ponerse en lugar de otro

Bien sea desarrollando la capacidad de escuchar o de interpretar una realidad diferente, el alumnado ha sido capaz de adquirir esta vertiente de la empatía. Se han puesto en su lugar y han comprendido las necesidades especiales que tiene una persona con diversidad funcional, descubriendo formas de comunicarse alternativas a las habituales. Igualmente han desarrollado la comprensión hacia la gente que realmente necesita ayuda:

"Nos han enseñado que no hacen falta palabras para comunicarse" (DSA-RF1) 
Todo ello genera un estado de satisfacción en el alumnado. Se sienten capaces de comprender los sentimientos y circunstancias ajenas difíciles y han apreciado que con su actuación han contribuido a una mejora en la calidad de vida de las personas receptoras del servicio:

"Nos ha gustado mucho ayudar a estos niños" (DSS-G11F1)

Relación con los demás

En la relación con otras personas destacan dos aspectos. Por una parte valorarlas y por otra tener una imagen positiva de ellas.

- Relación con los demás: valorar a los otros

Para un maestro o maestra ser capaz de relacionarse con los demás es una necesidad, ya que su desempeño profesional se basa en ello. Entendemos esta capacidad en una doble vertiente. Por una parte, se ha de ser capaz de valorar a los demás y por otra parte se ha de trasladar esa capacidad al ideario de actuación propio. Este es uno de los efectos del uso del ApS, que hemos codificado como "Lecciones aprendidas". En definitiva se trata de valores, actitudes y comportamientos que el alumnado ha identificado entre los receptores del servicio y que ha asumido como propios (capacidad de afrontar la realidad y los problemas derivados de la misma, superación, ejemplo a seguir, apreciar lo que se tiene, compartir sentimientos, comprensión y satisfacción). La siguiente cita ejemplifica estos resultados:

"Ver como uno de ellos recuerda tu nombre y lo dice en alto pese a no poder a penas controlar su cuerpo, o como una niña que apenas sabe decir su nombre, recuerda la melodía y sonríe al cantarle una canción. Todas estas cosas, que pueden parecer pequeñas, son reconfortantes. Son de esas, que cuando se acaba el día, te hacen sentir especial e incluso en paz contigo mismo." (DSA-RF3)

- Relación con los demás: tener una imagen positiva

Es importante tener una imagen positiva de los demás. Estimar en los otros la capacidad de esfuerzo y la alegría que transmiten a pesar de las dificultades son aprendizajes adquiridos por el alumnado.

"En conjunto hemos sentido rasgos de superación, esfuerzo, esperanza ya que tanto los educadores especializados, como los niños, como las fisios, han demostrado unas ganas inmensas de seguir adelante" (DSS-G12F1)

Vista esta exposición de datos, podemos decir que la capacidad de valorar a los demás y de tener una imagen positiva de ellos, son los aspectos que más se han desarrollado entre el alumnado con la aplicación del programa de ApS. Además lo valoran como una preparación necesaria para su futuro como docentes. 


\section{Consciencia social}

Esta subcategoría se desglosa en dos apartados: descubrimiento de la realidad social e implicación profesional.

\section{- Consciencia social: descubrimiento de la realidad social}

Las opiniones recogidas muestran que han descubierto un mundo que para ellos no existía (conciencia social): el de los colectivos de personas con diversidad funcional y con altas probabilidades de exclusión social. Es especialmente importante en la formación de un maestro o maestra desarrollar la capacidad de aprender de estas problemáticas y ser capaz de actuar ante ellas:

"Estas sesiones nos han servido para tomar contacto con un mundo diferente al que consideramos "normal" (DSS-G1F5)

- Consciencia social: implicación profesional

Hemos recogido referencias sobre sentimientos que experimentan al contactar y conocer personas con dificultades. Algunas reflexiones se limitan a su descripción, pero otras demuestran que se desarrolla un proceso de introspección, de reflexión, que ayuda a tomar conciencia de la realidad social en la que viven en contraposición con la que ellos y ellas conocen, propiciando la aparición de conductas y actitudes más implicadas socialmente. Asimismo destacan el valor formativo de las experiencias relatadas por las personas con diversidad sobre las ayudas que les prestan los profesionales que trabajan con ellos a diario ya que estas les sirven como modelo a seguir.

"Otro caso que nos emocionó, fue que el profesor de Educación Física de ese cole, cuando tiene a niños que no pueden caminar, a la hora de correr $o$ de ir en patines, les ha construido una caja de madera grande y abajo les ha puesto 4 ruedas para que ellos también puedan ir en patines, con la ayuda de él. Son cosas que nos han contado todo ellas, y que nos han impactado mucho." (DSA-RF11)

Realizado el análisis en la categoría de Sensibilidad Social, podemos afirmar que existen evidencias de que los participantes en el programa ApS han adquirido los componentes de esta categoría que hemos ido desgranando. El alumnado se muestra más sensible socialmente, lo que les puede ayudar a convertirse en mejores personas y docentes. Ser capaces de interpretar la realidad social en la que vivimos y de apreciar las dificultades de algunas personas les llevará a establecer en lo posible medidas correctoras en sus actuaciones diarias para eliminar la discriminación social. Formar estos aspectos entre estudiantes universitarios sólo puede propiciarlo la aplicación de metodologías como la que nos ocupa, siendo muy difícil trasladar estas vivencias a las aulas ordinarias. Categoría "Ayuda y colaboración” (AC)

Dentro de esta categoría hemos encontrado evidencias de que el alumnado ha desarrollado capacidades que les han permitido trabajar conjuntamente, compartiendo conocimientos, experiencias, esfuerzos y soluciones. Los hallazgos se dividen en dos subcategorías: colaboración alumnado/participantes y trabajo en grupo. 


\section{- Colaboración alumnado/participantes}

Las reflexiones demuestran la importancia de la colaboración existente tanto entre los propios receptores del servicio como entre estos y el grupo de trabajo. Este hecho puede determinar que en su futuro profesional el alumnado traslade con mayor ahínco el trabajo colaborativo a su ámbito de trabajo.

"Porque eso sí que lo notamos, que entre ellos se ayudaban mucho, y fue algo realmente gratificante de ver, porque se veía compañerismo en el ambiente" (DSS-G1F3)

\section{- Trabajo en grupo}

Participar, cooperar y colaborar en el trabajo común y construir soluciones por consenso son algunos de los aspectos que se pretendían fomentar entre el alumnado participante en el programa de ApS. Sin duda, se trata de conductas que tienen mucho que ver con la adquisición de hábitos de trabajo deseables en su futuro como maestros y como ciudadanos.

"La sesión ha sido realizada de manera cómoda ya que cada miembro aportaba muchas ideas y luego seleccionábamos las que más nos gustaban para proponérsela a los niños." (DSS-G7F5)

A la vista de estos resultados, podemos decir que el alumnado ha adquirido habilidades referentes al trabajo conjunto, al respeto hacia las opiniones de los demás, a la cooperación como forma de mejora grupal, a la implicación personal como forma de motivación de los demás y al desarrollo del compañerismo. Todo ello supone una mejora en la formación tanto profesional como personal.

\section{Categoría “Responsabilidad social” (RES)}

La responsabilidad social atiende a las siguientes particularidades o subcategorías: propia y profesional, familia y sistema educativo.

\section{- Propia y profesional}

La responsabilidad social es un concepto básico en la ciudadanía. El alumnado participante la ha adquirido, siendo capaz de interpretar su significado y de aplicarlo al autoimponerse determinadas tareas. Asimismo, la han percibido en la actuación de algunos profesionales de las entidades en las que han trabajado. Todo ello deriva en aprendizajes sociales relativos a la ética profesional.

"Las fisios que hay en el centro nos transmiten grandes ganas de hacer esfuerzos por los demás” (DSS-G12F1)

\section{- Sistema educativo}

También encontramos referencias a la responsabilidad social vinculada al sistema educativo. El alumnado indica que sólo con la cooperación y asumiendo cada parte su cuota de responsabilidad, el sistema educativo actuará correctamente: 
"la relación y el trabajo del maestro y de la familia debe ser coherente y cooperativa". (DSA-RF1)

\title{
- Familia
}

Otra visión de la responsabilidad la han adquirido a partir de la visión de los padres de los niños y niñas con diversidad funcional. Concretamente el alumnado ha entendido que es más responsable aceptar y afrontar la existencia de una dificultad que negarla o ignorarla.

\begin{abstract}
"Querríamos destacar una cosa que nos impactó mucho... sobre el niño XXXXX, bueno las fisios nos comentaron que este niño nunca podrá caminar, por lo tanto han hablado con sus padres para que entre todos le den una silla de ruedas mecánica para que el niño se pueda desplazar, y tenga una vida social más cómoda... Bueno, pues los padres les contestaron: mi hijo no necesita una silla de esas porque mi hijo camina.... Vale, comprendemos a los padres que siempre te queda esa esperanza de tu hijo, pero si los médicos dicen que nunca caminará es por algo, pues que menos que ayudarlo con una silla más cómoda" (DSS.G11F2)
\end{abstract}

Todos los aprendizajes sobre la responsabilidad social propiciados por esta experiencia resultarán útiles en el ejercicio futuro de su profesión.

\section{Discusión de resultados y conclusiones}

Billig (2000, 2002); Eyler, Giles, Stenson y Gray (2001) y Eyler y Giles (1999) categorizan los resultados de la aplicación del ApS en cuatro apartados: académicos, personales, sociales y de ciudadanía. Dado que hemos tomado como objeto de estudio el concepto "Competencia Social y Ciudadana", los resultados obtenidos en nuestra investigación podemos contrastarlos con estudios que hayan indagado en los resultados del uso de ApS en los tres últimos apartados de esta clasificación.

La metodología mixta utilizada descubre una concordancia entre los resultados obtenidos cuantitativa y cualitativamente. En ambas aproximaciones se observan mejoras en las dimensiones Ayuda y colaboración, Sensibilidad social, Conformidad con lo socialmente correcto y Responsabilidad social.

Las categorías Ayuda y Colaboración y Sensibilidad Social se corresponden con el apartado de resultados personales. Actualmente el individualismo es una de las actitudes predominantes en la sociedad. Cada cual se preocupa de uno mismo y de sus circunstancias ignorando las problemáticas de los demás, por lo que ayudar y colaborar con otros son valores que nos planteamos desarrollar con nuestra propuesta metodológica.. Los resultados nos muestran que son dimensiones adquiridas por nuestro alumnado. El ApS obliga al trabajo en grupo, más aún en un formato de servicio directo como el nuestro. Cada sesión debía estar perfectamente preparada y coordinada, entrañando lo contrario la asunción de un riesgo de no cumplir las expectativas, tanto ante los receptores como ante el personal profesional de las 
entidades. Trabajar así implica colaboración, integración, superación de dificultades en la interacción personal y llegar a consensos como señas de identidad en el funcionamiento de los grupos. El ApS en la modalidad de servicio directo aplicado en EF produce un "feedback" inmediato, ya que ante las contingencias se debe responder en tiempo real. El alumnado ha superado adecuadamente este hecho, desarrollando este sentido amplio de trabajo en grupo y los valores que lleva implícitos. Ha provocado mejoras en la comunicación, en el compañerismo y en la motivación ante el trabajo. Estos resultados coinciden con el trabajo de Billig, Root y Dan Jesse (2005) sobre los efectos de programas de ApS en la modalidad de servicio directo. Podemos apuntar, en consecuencia, que el ApS mejora los valores de colaboración, cooperación, integración y consenso en el trabajo en grupo. El programa de ApS ha permitido en el ámbito de la sensibilidad social que el alumnado haya mejorado su conciencia social, gracias al ejemplo proporcionado por los profesionales con los que han trabajado y descubierto una realidad social diferente a la cotidiana. Este estímulo puede cambiar sus actitudes en actuaciones futuras, bien sean personales o profesionales. Destacar la importancia del modelo que como docentes transmitimos a nuestro alumnado, siendo decisivo para su formación. Aplicar programas de ApS obliga a los docentes a ofrecer modelos de trabajo y de comportamiento diferentes a los tradicionales. Ante esta discusión, se apunta que el ApS produce mejoras en la conciencia social del alumnado. Llama la atención el desconocimiento del alumnado de la realidad social en la que viven. En este sentido, el uso del ApS en EF supone interacción del alumnado con la sociedad, provocando que conozcan la realidad y las necesidades existentes. La empatía es otra de las cuestiones que se ha conseguido acrecentar entre nuestros alumnos. Se observa que son capaces de ponerse en el lugar de otro, desarrollando diferentes estrategias de comunicación. Si un maestro/a carece de esta competencia, difícilmente desempeñará su labor eficazmente, y el ApS se muestra como una buena herramienta para ello.

La sensibilidad social lleva aparejada la capacidad de relacionarse con los demás de forma adecuada. Los resultados sugieren que el ApS ayuda a formar una imagen positiva de los demás y a valorarlos. Asimismo, el mundo de la EF es propicio para estos aprendizajes, ya que permite aflorar valores significativos para el alumnado.

Los resultados obtenidos en estas dos dimensiones coinciden con los descritos por Conway et al. (2009), quienes en una revisión determinaron mejoras limitadas en esta dimensión. Asimismo, Yorio y Feifei (2012) refieren mejoras en cuestiones de entendimiento social, conciencia y sensibilidad social y compromiso con las necesidades de la comunidad, aspectos que también concuerdan con los resultados de este trabajo.

La categoría Conformidad con lo Socialmente Correcto forma parte de los resultados sociales. Dado que la prestación del servicio se ha realizado dentro del mundo de la diversidad funcional y con menores de edad, el alumnado ha conocido y aprendido las normas legales y morales que rigen con estas personas a efectos de preservar su dignidad. Es un aprendizaje importante para su futuro laboral. En consecuencia, nuestros resultados siguen la misma tendencia que los reflejados por Conway et al. (2009). 
La Responsabilidad Social es una dimensión directamente relacionada con el concepto de ciudadanía activa. Comprender y ejercer la responsabilidad social es una cualidad fundamental que el alumnado debe desarrollar para ser competente como ciudadano. Con los datos obtenidos, se observa que el programa de ApS aplicado ha servido para concienciar al estudiantado sobre su propia responsabilidad social y la que tendrán como futuros docentes, comprendiendo además el protagonismo en cuanto a responsabilidad de otros dos actores del ámbito educativo: las familias y el sistema. Únicamente desde un funcionamiento integradoel sistema educativo podrá crear personas competentes socialmente. Conocer la responsabilidad social de todos los agentes implicados en el hecho educativo (familias, sistema y sociedad) es básico para ejercer una docencia comprometida, crítica y activa.

La cuestión de la participación activa es central en la competencia social y ciudadana. Es necesario implicarse en las estructuras y mecanismos que conforman el entramado social. La aplicación del programa ha sido positiva en el fomento de esta actitud. A la vista de los resultados, el alumnado ha entendido la capacidad de ayudar que posee y lo enriquecedor que es hacerlo. En este sentido, nuestra investigación presenta resultados alentadores alineados con los de Conway et al. (2009). En este sentido, también coincidimos con los resultados compilados por Yorio y Feifei (2012), quienes refieren mejoras importantes en la responsabilidad, habilidades interpersonales y valores éticos y morales, más similares a los mostrados por los estudiantes de nuestro estudio.

Este artículo presenta los resultados de aplicar un programa de ApS a estudiantes de maestro/a en el ámbito de la EF desde una perspectiva de investigación metodológica mixta. En concreto se analiza su incidencia en el desarrollo de la competencia social y ciudadana. La complementariedad cuantitativa-cualitativa nos ha ofrecido un marco adecuado de aproximación al objeto de estudio, en tanto que hemos obtenido una confluencia de resultados desde ambas perspectivas. Cuantitativamente hemos obtenido ciertas tendencias de mejora en algunas dimensiones que son refrendadas en el análisis cualitativo. En conclusión, a partir del objetivo planteado en la investigación y vistos los datos obtenidos y su discusión, podemos concluir que el ApS aplicado en el ámbito de la formación de futuros maestros y maestras es una buena herramienta para desarrollar su competencia social y ciudadana.

Respecto a las limitaciones y prospectiva para futuros trabajos sería deseable trabajar con una muestra más amplia y revisar la intensidad y duración del programa para asegurar la idoneidad del estímulo. De la misma forma, es necesario ir creando marcos de análisis de los efectos del ApS (estudiantes, receptores del servicio y comunidad) que ayuden a guiar futuros estudios e interpretar sus resultados en el contexto español. Asimismo, otra línea de trabajo debe ser la profundización en las tendencias obtenidas en este estudio en lo referente a los efectos del ApS en los estudiantes. 


\section{Referencias bibliográficas}

ANGUERA, M. T. (2011). Metodología cualitativa y cuantitativa, En C. Izquierdo y A. Perinat (Coords.), Investigar en psicología de la comunicación. Nuevas perspectivas conceptuales y metodológicas (pp. 209-230). Barcelona: Amentia.

ANGUERA, M.T., CAMERINO, O., CASTAÑER, M. y SÁNCHEZ-ALGARRA, P. (2014). Mixed methods en actividad física y deporte. Revista de Psicología del Deporte, 23 (1), 123-130

BARBOSA-RINALDI, I. (2008). Formação inicial em Educação Física: uma nova Epistemologia da prática docente. Movimento, 14, 185-207.

BILLIG, S. H. (2000). Research on K-12 school-based service learning: The evidence builds. Phi Delta Kappan, 81, 658-664.

BILLIG, S. H. (2002). Adoption, implementation, and sustainability of K-12 servicelearning, en Furco, A. y Billig, S.H. (eds) Advances in service-learning research: Vol.1. Service-learning: The essence of the pedagogy. Greenwich, CT., 245-267.

BILLIG, S. H., ROOT, S. y JESSE, D. (2005). The impact of participation in servicelearning on high school students' civic engagement. Circle Working Paper, 33. College Park, MD: Center for Information and Research on Civic Engagement and Learning.

BISQUERRA, R. y PÉREZ, N. (2007). Las competencias emocionales. Educación $X X 1,14,61-82$

CALVO-BERNARDINO, A. y MINGORANCE-ARNÁIZ, A.C. (2009). La estrategia de las universidades frente al Espacio Europeo de Educación Superior, Revista Complutense de Educación, 20(2), 319-342.

Cervantes, C.M. y Meaney, K.S. (2003). Examining Service-Learning Literature in Physical Education Teacher Education: Recommendations for Practice and Research, Quest, 65 (3), 332-353

CONWAY, J.M., AMEL, E.L. y GERWIEN, D.P. (2009). Teaching and Learning in the Social Context: A Meta-Analysis of Service Learning's Effects on Academic, Personal, Social, and Citizenship Outcomes. Teaching of Psychology, 36, 233 - 245.

CREMASCO, F. y CASTELLANI, L. (2012). Escola e Formação para a cidadania: qual o papel da Educação Física? Movimento,18, 135-154.

DOMANGUE, E., y Carson, R. (2008). Preparing Culturally Competent Teachers: Service-Learning and Physical Education Teacher Education. Journal of Teaching in Physical Education, 27(3), 347-367.

ESTEBAN, F. (2004). Excelentes profesionales y comprometidos ciudadanos. Un cambio de mirada desde la universidad. Bilbao: Desclée.

EYLER, J. y GILES, D. E. (1999). Where's the learning in service-learning? San Francisco: Jossey-Bass. 
EYLER, J.S., GILES, E.H., STENSON, M.C. y GRAY, CH. (2001). At a glance: What We Know about The Effects of Service-Learning on College Students, Faculty, Institutions and Communities, 1993-2000. Vanderbilt University, Corporation for National Service and Learn and Serve America National Service Clearinghouse. Recuperado de: http://www.compact.org/resources/downloads/aag.pdf. [consulta 2012, 12 de diciembre]

GARCÍA, R., ESCÁMEZ, J., MARTÍNEZ, M. y MARTÍNEZ, MJ. (2008). Aprendizaje de Ciudadanía y Educación Superior, en: Educación y Ciudadanía. Anroart Ediciones, S.L. Las Palmas de Gran Canaria.

GARCÍA, R., GOZÁLVEZ, V., VÁZQUEZ, V. y ESCÁMEZ, J. (2012). Repensando la Educación: cuestiones y debates para el siglo XXI. Valencia: Brief Ediciones.

Herrero, M. (2006). Ciudadanía y Universidad. Estudios sobre Educación, 10, 155-173.

HIMELEIN, M., PASSMAN, L., y PHILLIPS, J. M. (2010). College Teaching and Community Outreaching: Service Learning in an Obesity Prevention Program. American Journal of Health Education, 41(6), 368-378.

LAMASTER, K. J. (2001). Enhancing Preservice Teachers Field Experiences through the Addition of a Service-Learning Component. Journal of Experiential Education, 24(1), 27-33.

MARTÍNEZ, M. (2006). Formación para la ciudadanía y educación superior. Revista Iberoamericana de Educación, 42, 85-102.

MASSEY-SOKES, M., y MEANEY, K. S. (2006). Understanding Our ServiceLearning Community: An Exploratory Study of Parent, Teacher, and Student Perceptions about Childhood Obesity. Health Educator, 38(2), 53-60.

Moraleda, A., González, J. y García-Gallo, J. (2004). AECS, actitudes y estrategias cognitivas sociales. Madrid: TEA ediciones.

PALOMARES, A. (2011). El modelo docente universitario y el uso de nuevas metodologías en la enseñanza, aprendizaje y evaluación. Revista de Educación, 355, 591-604.

PAGÈS, J. (2009). Competencia social y ciudadana. Aula de Innovación Educativa, 187, 7-11.

PENALVA, J. (2009). Paradigmas escolares vigentes. Influjos en el sistema educativo español. Educación XX1, 12, 181-199.

PRINT, M. (2003). Estrategias de enseñanza para la educación cívica y ciudadana en el siglo XXI. Estudios sobre Educación, 4, 7-22.

PUIG, J. M., BATLLE, R., BOSCH, C. y PALOS, J. (2007). Aprendizaje servicio. Educar para la ciudadanía. Barcelona: Octaedro.

PUIG, J.M., GIJÓN, M., MARTÍN, X. y RUBIO, L. (2011). Aprendizaje-servicio y Educación para la Ciudadanía. Revista de Educación, número extraordinario 45-67. 
RMC RESEARCH CORPORATION. (2006). K-12 Service-Learning Project Planning Toolkit. Scotts Valley, CA: National Service-Learning Clearinghouse. Recuperado de: http://www.servicelearning.org/filemanager/download/K-12_Service-Learning Project_Planning_Toolkit.pdf. [consulta 2012, 14 de diciembre]

RIERA, J. y CIVÍS, M. (2008). La pedagogía profesional del siglo XXI. Educación XX1, 11, 133-154.

RÍOS, M. (2003). El juego y el alumnado con discapacidad intelectual y/o plurideficiencia.Tándem: Didáctica de la Educación Física, 4(11), 40-49.

RÍOS, M. (2009). La Educación Física adaptada a pacientes con trastorno mental severo: una experiencia de aprendizaje servicio. Tándem: Didáctica de la Educación Física, 8(29), 79-90.

RODRÍGUEZ, M. R. (2014). El Aprendizaje-Servicio como estrategia metodológica en la universidad. Revista Complutense de Educación, 25, 95-113.

SANTOS, M.A. (2013). ¿Para cuándo las universidades en la agenda de una democracia fuerte? Educación, aprendizaje y compromiso cívico en Norteamérica. Revista de Educación, 361, 565-590.

SANTOS, M. A. y LORENZO, M. (2012). Dimensión cívica y desarrollo formativo de los estudiantes universitarios en el contexto español. Revista Electrónica de Investigación Educativa Número Especial. Recuperado de: http://redie.uabc.mx/NumEsp2/contenido-rego.html [consulta 2012, 14 de diciembre]

TOURIÑÁN, J.M. (2007). Valores y convivencia ciudadana: una responsabilidad de formación compartida y derivada. Bordón, 59, 261-311.

VEGA, A. (2007). De la dependencia a la autonomía: ¿dónde queda la educación?. Educación XX1,10, 239-264.

YORIO, P. L. Y FEIFEI, Y. (2012). A meta-analysis on the effects of ServiceLearning on the social, personal, and cognitive outcomes of learning. Academy of Management Learning \& Education, 11, 9-27. 


\section{Correspondencia con los autores}

Jesús GIL-GÓMEZ

Facultad de Ciencias Humanas y Sociales

Departamento de Educación

Universitat Jaume I

Av. de Vicent Sos Baynat, s/n

12071 Castellón de la Plana, España

e-mail: jegil@uji.es

Odet MOLINER-GARCÍA

Facultad de Ciencias Humanas y Sociales

Departamento de Educación

Universitat Jaume I

Av. de Vicent Sos Baynat, s/n

12071 Castellón de la Plana, España

e-mail:molgar@uji.es

Óscar CHIVA-BARTOLL

Facultad de Magisterio

Departamento de Didática de la expresión musical, plástica y corporal

Unidad docente de Educación Física

Universitat de València

Avda. Tarongers, 4

46022 València.

Apartado de Correos 22045 -

46071 Valencia, España.

e-mail: oscar.chiva@uv.es

Rafaela GARCÍA LÓPEZ † 